\title{
1 Rapid detection of Salmonella using a redox cycling-based electrochemical 2 method
}

\author{
3 Danhui Wang ${ }^{\mathrm{a}}$, Ziyuan Wang ${ }^{\mathrm{a}}$, Juhong Chen ${ }^{\mathrm{a}}$, Amanda J. Kinchla ${ }^{\mathrm{a}}$, Sam R. Nugen ${ }^{\mathrm{a}^{*}}$ \\ $4 \quad$ a Department of Food Science, University of Massachusetts, Amherst, MA 01003 \\ $5 \quad$ *corresponding author: snugen@umass.edu
}

\begin{abstract}
An electrochemical method based on redox cycling combined with immunomagnetic separation and pre-concentration was developed for rapid and sensitive detection of Salmonella. Electrochemical methods for the detection of bacteria offer the advantages of instant quantification with minimal equipment. Unfortunately, the limits of detection are often poor compare to other transduction methods such as fluorescence and chemiliuminescence. We demonstrated an electrochemical method which is both rapid and has a low limit of detection. A two-step strategy, which included immunomagentic pre-concentration and redox cycling was used to amplify the signal. Magnetic beads modified with antiSalmonella antibodies were used for separation and pre-concentration of Salmonella from phosphate buffered saline (PBS) and agricultural water. Then anti-Salmonella antibodies conjugated with alkaline phosphatase were employed for labeling the Salmonella which had been captured by magnetic beads. Alkaline phosphatase (ALP) catalyzed the substrate Lascorbic acid 2-phosphate (AAP) to electroactive species L-ascorbic acid (AA) while tris(2-carboxyethyl)phosphine (TCEP) facilitated the regeneration of AA on the gold electrode to form redox cycling resulting in an amplified signal. Under the optimal conditions, the Salmonella in PBS buffer as well as in agricultural water were detected. The limit of detection of this approach was approximately $7.6 \times 10^{2} \mathrm{CFU} / \mathrm{mL}$ and $6.0 \times 10^{2} \mathrm{CFU} / \mathrm{mL}$ in PBS buffer and agricultural water, respectively, without pre-enrichment in 3 hours. When the agricultural water has been pre-enriched for 4 hours, the limit of detection was approximately $10 \mathrm{CFU} / \mathrm{mL}$.
\end{abstract}

Keywords: biosensor, food pathogen, rapid detection

\section{Introduction}

Salmonella continues to be one of the most serious pathogens in food and agriculture (Shim, Song, Mun, Chung, \& Kim, 2014). Infections resulting from this pathogen can be fatal and its ubiquity has caused it to be a worldwide health concern (Lee, Runyon, Herrman, Phillips, \& Hsieh, 2015; L. J. Yang, Ruan, \& Li, 2001). The low infective dose required for infection makes control of the pathogen difficult and scores the need for strict monitoring (Liebana, et al., 2009). Therefore, it is essential to selectively and sensitively detect Salmonella in order to provide adequate food safety assurance. Conventional methods based on microbiological techniques such as swabbing, pre-enrichment and selective plating are the most accurate and reliable approaches for the assay detecting pathogens and are the standard methods used by regulatory agencies (Cho, Mauer, \& Irudayaraj, 2014; Velusamy, Arshak, Korostynska, Oliwa, \& Adley, 2010). However, most conventional techniques which involve the pre-enrichment with subsequent colony counting can take days for results (Shim, et al., 2014; Taskila, Tuomola, \& Ojamo, 2012; Yeni, Acar, Polat, Soyer, \& Alpas, 2014). The limitation of these approaches is the long assay time, complicated preparation procedures and labor intensiveness. Thus, there is increased demand to develop strategies for a rapid, sensitive and accurate detection for Salmonella in food matrix (Nugen \& Baeumner, 2008). In recent decades, numerous efforts have been made to develop rapid screening methods to provide an initial level of assurance to the food manufacturer. 
In order to increase the detection sensitivity, several possible pre-concentration steps have been employed prior to performing an assay (L. J. Yang, et al., 2001). Immunomagnetic separation (IMS) is a rapid and specific approach to separate and concentrate bacteria from a liquid food matrix which offers several advantages, using magnetic beads conjugated with specific target antibody which are used to selectively capture, separate and concentrate bacteria or other analytes from a complex sample. It is able to increase the efficiency of separation and concentration of bacteria and has a high selectivity to specific bacteria compared with centrifugation and membrane filtration(Chen, Alcaine, Jiang, Rotello, \& Nugen, 2015; Shields, et al., 2012; H. Wang, Li, Wang, \& Slavik, 2011).The immunomagnetic assay is commonly coupled with colorimetric or electrochemical methods. However, the colorimetric method has limitation in testing turbid samples compared with electrochemical method. Because the results were hard to accurately reported as the turbidity interfered with the readings(Liebana, et al., 2009). In this study, immunomagnetic assay was combined with a rapid, ultrasensitive electrochemical detection method.

Electrochemical detection has emerged as a potential alternative to avoid the limitations of conventional methods (Arora, Chand, \& Malhotra, 2006; Chen, Zhou, et al., 2015) and has attracted particular interest due to its advantages such as low equipment costs, portability, instant quantification, and short detection time (Chen, Jiang, et al., 2015; Guosong Lai, 2009). Electrochemical detection is conducted by detecting an electroactive compound generated on the electrode that usually catalyzed by an enzyme. However, single enzyme label is restricted by the amount of the enzyme substrate in which an antibody conjugated with an enzyme when employed for the capture of target and generation of electroactive species (Nam, et al., 2012). In order to get ultra-low limit of detection, the strategy for signal amplification can be critical for electrochemical detections. There have been several versatile approaches based upon chemical or physical amplification attempted, such as multienzyme and redox cycling (Wilson, 2004). Therefore, in order to increase the signal, redox cycling was used as an additional method for amplification coupled with enzymatic reaction in this research.

The enzyme-based detection plus redox cycling has arisen more attention on it because of its ultra-sensitivity and simplicity. This method requires the addition of reactants to common enzyme-based detection methods (Walter, Wu, Flechsig, Haake, \& Wang, 2011; Xia, et al., 2013; H. Yang, 2012). Redox cycling is a process where the electroactive species are regenerated either with the use of an additional electrode or chemical. The addition of tris(2carboxyethyl)phosphine (TCEP), an oxidation-resistant water-soluble reductant has been shown to provide a significant amplification to electrochemical detection by reducing the electrode oxidized reactant and thereby enabling it to repeat the oxidation cycle at the electrode ( $\mathrm{Wu}, \mathrm{Yan}, \mathrm{Yan}, \& \mathrm{Ju}, 2008$ ). Further development and extensive application of this method relies on the choice of suitable enzyme and substrate. Alkaline phosphatase (ALP) is an enzyme that is commonly used as a label in the colorimetric or electrochemical assays. In electrochemical detection, it is able to catalyze many phosphate substrates with a low electric potential in short incubation periods thereby producing electroactive species. These properties make alkaline phosphatase an ideal enzyme label for redox-based electrochemical detection (Md Rajibul Akanda, et al., 2011; H. Yang, 2012).

In our study, L-ascorbic acid 2-phosphate (AAP) was selected as the substrate based on previous research. Here, signal amplification was obtained using ALP as an enzyme label and TCEP to form the redox cycling. Salmonella enteric was captured and pre-concentrated from either PBS buffer or agricultural water using magnetic beads conjugated to antiSalmonella antibodies. The captured bacteria were then mixed with anti-Salmonella-ALP which allowed an electrochemical detection. The enzyme-based electrochemical detection is based on electrochemical redox cycling, in which ALP acts as an enzyme, AAP as an enzyme substrate and TCEP as a reducing agent (Singh, 2013). It was essential to optimize the incubation time, temperature and reaction $\mathrm{pH}$ for the enzyme reaction in order to achieve a lower limit of detection. The key factors that affected the electrochemical reaction were investigated. The objectives of this research were to develop a more rapid and sensitive approach for bacterial detection, and to evaluate its application in agricultural water samples. Following optimization of the enzymatic reaction, a detection limit for Salmonella was obtained. 


\section{Material s and Methods}

\subsection{Bacterial culture samples}

Salmonella enterica subsp. (ATCC 14028) was obtained from the American Type Culture Collection (ATCC, VA, USA) and stored in $-80^{\circ} \mathrm{C}$ fridge. The pure culture of Salmonella was grown at $37^{\circ} \mathrm{C}$ overnight $(18-20 \mathrm{~h})$ in $50 \mathrm{~mL}$ of LB broth (Lysogeny broth, $10.0 \mathrm{~g}$ tryptone, $5.0 \mathrm{~g}$ yeast extract. $10.0 \mathrm{~g} \mathrm{NaCl}$ in $1 \mathrm{~L}$ distilled water) in an agitating incubator (Thermo Scientific, MA, USA). Approximately $10^{9} \mathrm{CFU} / \mathrm{mL}$ Salmonella were obtained from the incubation. Ten-fold serial dilutions of the overnight pure culture were prepared in $1 \times$ PBS buffer (phosphate buffered saline, $\mathrm{pH} 7.2,137.0 \mathrm{mM}$ $\mathrm{NaCl}, 2.7 \mathrm{~m} \mathrm{MKCl}, 10.0 \mathrm{mM} \mathrm{Na}_{2} \mathrm{HPO}_{4}, 2.0 \mathrm{mM} \mathrm{KH}_{2} \mathrm{PO}_{4}$ ) and the concentration of Salmonella was determined by plating $0.1 \mathrm{mLof}$ the dilutions on HE agar (Hektoen enteric, BD ${ }^{\mathrm{TM}}$, MD, USA). Another five milliliter of the original culture was heated for $15 \mathrm{~min}$ at $90{ }^{\circ} \mathrm{C}$ to denature the somatic antigens. The ALP conjugated anti-Salmonella antibodies are able to bind with both the live cells and heat-inactivated cells. Previous reports have suggested that the binding efficiency is higher for inactivated cells than live cells (Y. Liu, 2001). This is most likely due to an improved epitope availability upon heating. Therefore, in the proposed assay Salmonella cells were heat-treated prior to capture. The sample containing Salmonella was then serially diluted to approximately $10^{2}, 10^{3}, 10^{4}$ and $10^{5} \mathrm{CFU} / \mathrm{mL}$ in $10 \mathrm{~mL} 1 \times$ PBS buffer (pH 7.2). All other chemicals were obtained from Fisher Scientific (Fairlawn, NJ, USA).

\subsection{Agricultural water samples}

Two sources of agricultural water were obtained from two locations five miles apart in the Connecticut River in Western Massachusetts. This river is a common source of agricultural water for farms in the area. The agricultural water had been confirmed as no Salmonella originally by plating on HE agar before using. The agricultural water samples were prepared by serially diluting the heated Salmonella to approximately $10^{2}, 10^{3}, 10^{4}, 10^{5} \mathrm{CFU} / \mathrm{mL}$ in $10 \mathrm{~mL}$ agriculture water and then were used in the electrochemical detection.

\subsection{Immunomagnetic pre-concentration of Salmonella}

Aliquots containing $50 \mu \mathrm{L}$ of anti-Salmonella magnetic particles (Dynabeads $\AA$, Invitrogen ${ }^{\mathrm{TM}}$, Life Technologies, Norway) were added into $10 \mathrm{~mL}$ Salmonella samples $\left(0,10^{2}, 10^{3}, 10^{4}\right.$ or $\left.10^{5} \mathrm{CFU} / \mathrm{mL}\right)$. The anti-Salmonella magnetic beads are able to capture all current Salmonella serovars which are the main cause of human and animal disease associated with environmental, feed and food samples according to the manual and protocol of the product. The capture efficiency (CE) of the immunomagnetic beads was determined and the CE was over $80 \%$ which has reached a satisfied level (Supplementary information). This bead concentration has been previously demonstrated to be sufficient for the capture of Salmonella in 30 minutes (Steingroewer, Knaus, Bley, \& Boschke, 2005; Tatavarthy, et al., 2009; Zheng, Mikg-Krajnik, Yang, Xu, \& Yuk, 2014). The mixture was then agitated on a carousel mixer at room temperature for 30 minutes at 20 RPM to prevent beads from settling. Following agitation, the tubes were inserted into a strong cell separation magnet $\left(\mathrm{BD}\right.$, IMagnet $\left.{ }^{\mathrm{TM}}\right)$ and allowed 3 minutes for proper recovery of beads and concentration of the conjugates to the side wall of the tube from the suspension. Three washing steps of the concentrated beads were performed using $1 \mathrm{~mL}$ of $1 \times$ PBS with $0.1 \%$ (w/v) bovine serum albumin (BSA). The supernatant was then discarded and the conjugates were resuspended in $1 \mathrm{~mL}$ of $1 \times \mathrm{PBS}$ and transferred to $2 \mathrm{~mL}$ microcentrifugetube. Twenty microliters of anti-Salmonella antibodies conjugated with alkaline phosphatase $(0.01 \mathrm{mg} / \mathrm{mL}, \mathrm{KPL}, \mathrm{MD}$, USA) were added to the mixture and incubated with agitation for 30 min at room temperature. Three washing steps were performed using TBS (Tris-buffered saline, $\mathrm{pH}$ 8.0, $25 \mathrm{mM}$ Tris, $150 \mathrm{mM} \mathrm{NaCl}$ ) with $0.1 \%(\mathrm{w} / \mathrm{v})$ BSA. Finally, the conjugates were resuspended in $20 \mu \mathrm{L}$ TBS (Tris-bufferred saline, $\mathrm{pH} 10.0,25 \mathrm{mM}$ Tris, $150 \mathrm{mM} \mathrm{NaCl})$.

\subsection{Electrochemical detection}


The antibody-tagged alkaline phosphatase which was used to label the Salmonella was detected electrochemically using a prolonged redox reaction on a gold electrode. In the enzyme reaction, AAP was used as a substrate to produce the electroactive compound AA which could be electrochemically detected on the electrode. The reaction and subsequent signal generation resulted in the oxidation of AA at the electrode surface. The oxidized AA was then reduced back into the electroactive form of AA by the reductant TCEP allowing additional signal generation at the electrode surface (Figure 1). TCEP was selected as the reductant due to its stability and wide range of $\mathrm{pH}$ tolerance. The AA in the sample solution was able to cycle continuously until the TCEP was consumed which resulted in an electrochemical amplification. The concentration of AA was directly proportional to the concentration of ALP in the sample.

Following the immunomagnetic separation and ALP labeling of the Salmonella, $80 \mu \mathrm{L}$ of the AAP and TCEP in Tris buffer (50mMTris, pH 9.6) was added into sample. The solution was then incubated for 30 minutes at $40{ }^{\circ} \mathrm{C}$ to allow for the conversion of AAP to AA. Following the initial enzymatic reaction, $20 \mu \mathrm{L}$ of solution was transferred onto a gold electrode (MicruX Technologies Austurias, Spain) connected to a potentiostat. An electrochemical Potentiostat/ Galvanostat/ ImpedenceAnalyser (PalmSens, Netherlands) was used for all measurements using a potential of $0.6 \mathrm{~V}$. The charge (coulombs) was measured using the following conditions: Time for equilibration: 3s; Potential applied: 0.6V; Interval time: $0.1 \mathrm{~s}$; Run time: $200 \mathrm{~s}$. Chronocoulometry was conducted for the detection which is the measurement of the charge generated on the electrode per unit time $(\mathrm{C} / \mathrm{s})$ and is commonly used in electrochemical detection (Anson \& Osteryoung, 1983; Park \& Yang, 2014). Chronocoulograms (the charge versus time) were obtained for each detection. The charge at $150 \mathrm{~s}$ was used as the signal for the variables. The gold electrode was washed using $0.1 \mathrm{M} \mathrm{H}_{2} \mathrm{SO}_{4}$ by cyclic voltammetry in the range of $-1.0 \mathrm{~V}$ and $1.0 \mathrm{~V}$ after each individual measurement to allow for cleaning.

Although the optimal reaction conditions of alkaline phosphatase have been widely studied (Kreuzer, O'Sullivan, \& Guilbault, 1999; Mazzei, et al., 2004), it is known that immobilization and/or conjugation of an enzyme to an antibody can influence these optimal conditions (Goddard \& Hotchkiss, 2007). Therefore, in order to achieve a lower limit of detection, the effect of time, $\mathrm{pH}$ and temperature on the ALP reaction rate with AAP was determined. The incubation time was varied from 0 to 50 minutes. The $\mathrm{pH}$ of the substrate solution was adjusted using $1 \mathrm{M} \mathrm{HCl}$ or $\mathrm{NaOH}$ to $\mathrm{pH} 6.0,7.0$, 8.0, 9.0, 10.0, 11.0 and 12.0. The incubation temperature was varied by water bath to $20^{\circ} \mathrm{C}, 30^{\circ} \mathrm{C}, 40^{\circ} \mathrm{C}, 50^{\circ} \mathrm{C}$ and $60^{\circ} \mathrm{C}$. Additionally, the concentrations of AAP and TCEP in the reaction mixture were optimized for an increased signal to noise ratio. The concentrations investigated were $1.0 \mathrm{mM}$ AAP/2.0 mM TCEP; $5.0 \mathrm{mM}$ AAP/5.0 mM TCEP; $5.0 \mathrm{mM}$ AAP/10.0 mM TCEP; 10.0 mM AAP/5.0 mMTCEP; 10.0 mM AAP/10.0 mM TCEP; and 20.0 mM AAP/10.0 mM TCEP.

\subsection{Pre-enrichment of inoculated Salmonella in agricultural water}

Agricultural water $(44.5 \mathrm{~mL})$ was mixed with $5 \mathrm{~mL}$ of $10 \times \mathrm{LB}$ broth in $150 \mathrm{~mL}$ sterile flask and $0.5 \mathrm{~mL}$ of a known concentration of Salmonella culture (approximately $10^{3} \mathrm{CFU} / \mathrm{mL}$ ) such that the final sample contained a concentration of approximately $10 \mathrm{CFU} / \mathrm{mL}$ of Salmonella which was confirmed by plating. Following incubation of the broth $(1 \mathrm{~h}, 2 \mathrm{~h}, 3 \mathrm{~h}$, $4 \mathrm{~h}$, and $5 \mathrm{~h}$ ), the concentration of Salmonella was enumerated on HE agar using a 24 hour incubation at $37^{\circ} \mathrm{C}$.

\subsection{The specificity of the detection}

One hundred microliter of approximately $10^{6} \mathrm{CFU} / \mathrm{mL}$ of each four bacteria strain, including Salmonella enterica, E .coli BL 21, Staphylococcus aureus (S. aureus) and Pseudomonas aeruginosa (P. aeruginosa), was inoculated in agricultural water to achieve a final concentration of $10^{5} \mathrm{CFU} / \mathrm{mL}$ for each strain. The sample with $0 \mathrm{CFU} / \mathrm{mL}$ of bacteria was used as the negative control. Twenty microliter of immunomagnetic beads coated with anti-Salmonella antibody was added into 1 $\mathrm{mL}$ of each sample and incubated for $30 \mathrm{~min}$ at room temperature on a rotator. After incubation and washing for three times, twenty microliter of anti-Salmonella conjugated with ALP was added into the tubes and incubated for another 30 min at room temperature. After washing for three times, the conjugates were used for electrochemical detection following the steps in section 2.4. The charge signals were obtained for each group. 


\subsection{Statistical analysis}

The one-way analysis of variance (ANOVA, SAS Institute, Inc., Cary, NC) was conducted to calculate the mean values and standard deviations and evaluate the difference between the agricultural water from two locations. In comparison, mean values among treatments were determined significantly different at $\mathrm{P}<0.05$ with pooled-t-test. The data presented represents a mean of a minimum of three independent samples and error bars represent the standard deviation of the replicates.

\section{Results and Discussion}

\subsection{The optimization of enzyme reaction}

Several variables such as temperature, time and $\mathrm{pH}$ will have a significant effect on an enzymatic reaction. In order to achieve a lower limit of detection, it is necessary to optimize the condition of the enzyme reaction to ensure the optimal activity of ALP. The ratio of AAP and TCEP, reaction time, temperature and $\mathrm{pH}$ were varied to find the optimal condition for the reaction.

\subsubsection{The concentration of AAP and TCEP}

It was necessary to optimize the concentration of AAP and TCEP which were used in the enzyme reaction in order to achieve a higher signal-to-background ratio. These optimization reactions were carried out by incubating $20 \mu \mathrm{L}$ of the alkaline phosphatase-tagged antibody $\left(5 \times 10^{-5} \mathrm{mg} / \mathrm{mL}\right)$ with $80 \mu \mathrm{L}$ of varying concentrations of AAP and TCEP in Tris buffer $(50 \mathrm{mM}, \mathrm{pH} 10.0)$. Because changes in concentration of AAP and TCEP may have different electric potentials on the electrode, it was necessary to measure the signal without the ALP as well. The signal:background ratio was calculated after measuring the signal of varying concentrations of AAP and TCEP with and without ALP.

Figure 2a shows the charge at 150 seconds with different concentration of AAP and TCEP in the presence and absence of ALP. From Figure 2a, it was demonstrated that the signal generally increased with increasing concentrations of AAP and TCEP both with and without enzyme. Although it is essential to obtain a high signal for the enzyme reaction, it is also important to maintain a low background when the limit of detection was determined. It can be seen at $5 \mathrm{mM}$ or $10 \mathrm{mM}$ AAP, an increase of TCEP concentration from $5 \mathrm{mM}$ to $10 \mathrm{mM}$ resulted in an increased signal in the presence of ALP while little change was observed with no ALP. This suggests the increased signal was due to the additional redox cycling provided by the TCEP. Figure 2bdemonstrates the calculated signal-to-background ratios using the data from Figure $2 \mathrm{a}$. The combination of $5 \mathrm{mM}$ AAP and $10 \mathrm{mM}$ TCEP was used for subsequent experiments in order to allow for an increased signal:noise ratio.

\subsubsection{Incubation time}

The reaction time was first investigated at room temperature in Tris buffer ( $\mathrm{pH} 8.9)$, where AAP, TECP and ALP were incubated together for $5 \mathrm{~min}, 10 \mathrm{~min}, 15 \mathrm{~min}, 20 \mathrm{~min}, 25 \mathrm{~min}, 30 \mathrm{~min}, 40 \mathrm{~min}$ and $50 \mathrm{~min}$, respectively. Twenty microliters of alkaline phosphatase-tagged antibodies $\left(5 \times 10^{-5} \mathrm{mg} / \mathrm{mL}\right)$ were mixed with $80 \mu \mathrm{L}$ of a solution containing $5 \mathrm{mM}$ AAP and 10mM TCEP. The relatively low concentration of antibodies was selected to be similar to those which would be found with low Salmonella concentrations in the final assay. Following incubation, the sample was then placed on a gold electrode and the charge was determined as outlined in section 2.4.

It can be observed that the generated charge of AA increased gradually with increasing incubation time up to approximately 30 minutes. After 30 minutes, the charge increased slightly over the next 20 minutes (Figure 3a). An incubation time of 30 minutes was used for subsequent reactions in order to allow for optimal conversions of AAP to AA prior to the electrochemical detection. 


\subsubsection{Incubation temperature}

Temperature is another critical factor which affects enzyme reactions. In order to optimize the conversion of AAP to AA, the activity of ALP with respect to temperature was determined. Incubation temperatures of $20{ }^{\circ} \mathrm{C}, 30^{\circ} \mathrm{C}, 40^{\circ} \mathrm{C}, 50{ }^{\circ} \mathrm{C}$, and $60^{\circ} \mathrm{C}$ were investigated to determine the optimal conditions. Figure $3 \mathrm{~b}$ shows how the incubation temperature of the solution (5 mM AAP and $10 \mathrm{mM}$ TCEP in Tris buffer, $50 \mathrm{mM}, \mathrm{pH} 8.9$ ) affected the charge obtained on the electrode at 150 seconds when reacting with alkaline phosphatase-tagged antibodies $\left(5 \times 10^{-5} \mathrm{mg} / \mathrm{mL}\right)$. The results demonstrated that the charge was optimal at approximately $40{ }^{\circ} \mathrm{C}$. Therefore, an incubation temperature of $40{ }^{\circ} \mathrm{C}$ was used for all subsequent electrochemical reactions.

\subsubsection{Reaction pH}

Alkaline phosphatase has a reported optimal pH range being 8.0 - 10.0 (Dean, 2002). High pH values have been found necessary for optimal activity of alkaline phosphatase, but the $\mathrm{pH}$ optimum may vary depending on substrate and substrate concentration. In order to determine the $\mathrm{pH}$ dependence of the ALP-tagged antibody, 5.0 mM AAP and 10.0 mM TCEP were mixed together and adjusted to a $\mathrm{pH}$ of 6.0, 7.0, 8.0, 9.0 10.0, 11.0 and 12.0 in Tris buffer $(50 \mathrm{mM})$. The charge from the electrochemical reaction was determined for each of these $\mathrm{pH}$ conditions. Following the addition of the ALP-tagged antibodies, the sample was incubated at $40{ }^{\circ} \mathrm{Cfor} 30$ minutes. The electrochemical detection was then determined as outlined in section 2.4. It can be observed that from the Figure 3c the current increased with the $\mathrm{pH}$ increased from $\mathrm{pH} 6.0$ to $\mathrm{pH} 10.0$ and then decreased from $\mathrm{pH} 10.0$ to $\mathrm{pH} 12.0$ which indicated that the optimal $\mathrm{pH}$ was approximately 10.0 .

In the initial experiments, the optimal incubation conditions and AAP/TCEP concentrations for the antibody-tagged ALP were determined. For all subsequent electrochemical tests, an incubation condition of 30 minutes, $40{ }^{\circ} \mathrm{C}$ and $\mathrm{pH} 10.0 \mathrm{was}$ used. The concentration of AAP and TCEP used for subsequent experiments was $5.0 \mathrm{mM}$ and $10.0 \mathrm{mM}$, respectively.

\subsection{Electrochemical detection of Salmonella}

The electrochemical detection was based on redox cycling using TCEP as a reducing agent, in which alkaline phosphatase catalyzed the substrate AAP into electroactive species AA. TCEP, a strong reductant, is able to help regeneration of AA and prevent the auto-oxidization of AA. After the accumulation of AA for the sufficient time at optimal $\mathrm{pH}$ and temperature, the charge (coulomb) of the AA on the gold electrode was measured with a potential of $0.6 \mathrm{~V}$. The charge data were obtained at $150 \mathrm{~s}$ from the chronocoulograms at varying concentrations of Salmonella in both buffer and agricultural water. Agricultural water is commonly used to irrigate or rinse the agricultural products. Vegetables and fruits can be contaminated if there is foodborne pathogen in the agricultural water. The bacteria like Salmonella can attach to the surface firmly or internalize within it, so the agricultural products may cause the health issue for consumers if they are treated with contaminated agricultural water. Therefore, we applied this strategy in detection Salmonella in agricultural water. The magnitude of the charge was proportional to the amount of formed electroactive species AA on the electrode and the concentration of Salmonella in the sample matrix. Thus, the concentrations of Salmonella in the matrix could be determined by measuring the corresponding charge in a short time.

After the conditions of the enzyme reaction were optimized, the electrochemical detections for serially diluted samples of Salmonellain $1 \times$ PBS buffer and agricultural water were performed, respectively. Figure 4a shows the charge data for Salmonella at concentrations of $0,4 \times 10^{2}, 4 \times 10^{3}, 4 \times 10^{4}, 4 \times 10^{5} \mathrm{CFU} / \mathrm{mL}$. For all variants, the charge was relatively linear after approximately 30 seconds. This is most likely due to the time required for the redox cycling reached a steady state (M. R. Akanda, et al., 2013). The charges at 150s were plotted to determine the limit of detection. Figure $4 \mathrm{~b}$ and $4 \mathrm{c}$ representthe results of this electrochemical detection based on the charge recorded at $150 \mathrm{~s}$ for Salmonella inoculated in $1 \times$ PBS buffer and agricultural water, respectively. All data obtained were subtracted by the average value of background $(0 \mathrm{CFU} / \mathrm{mL}$ ).We can observe that the charge directly correlated to the concentration of Salmonella in matrix. The estimated detection limit of the electrochemical detection for Salmonella in $1 \times$ PBS buffer and agricultural water was 
$7.6 \times 10^{2}$ and $6.0 \times 10^{2} \mathrm{CFU} / \mathrm{mL}$, respectively. The limit of detection (LOD) was estimated by the comparison of the mean value of negative control plus three standard deviations and the corresponding Salmonella concentration (Kessler, 1998; Kjems, et al., 1993; Y. Wang, Fill, \& Nugen, 2012). The time required for the electrochemical detection was relatively short and thus this electrochemical approach was able to detect Salmonella in PBS buffer and agricultural water inoculated with Salmonella in 3 hours.

Furthermore, the pre-enrichment step was conducted to estimate the time for Salmonella growing from $10 \mathrm{CFU} / \mathrm{mL}$ to $10^{3}$ $\mathrm{CFU} / \mathrm{mL}$ in the mixture of LB broth and agricultural water. Pre-enrichment steps are commonly added to increase the concentration of viable bacterial cells. During this time, non-viable (heated) cells would remain in low concentrations and therefore not detected. As a result, the time for Salmonella growing from $10 \mathrm{CFU} / \mathrm{mL}$ to $10^{3} \mathrm{CFU} / \mathrm{mL}$ was approximately 4 hours which reached the limit of detection of this method. Therefore, after a pre-concentration of Salmonella for 4 hours and electrochemical detection procedure for 3 hours, we are able to detect approximately $10 \mathrm{CFU} / \mathrm{mL}$ of Salmonella in agricultural water.

The specificity of the proposed detection strategy was performed by using a mixture of four different bacteria strains, including Salmonella enterica, E .coli BL 21, Staphylococcus aureus (S. aureus) and Pseudomonas aeruginosa (P. aeruginosa). The concentration of each bacteria strain in the single or mix sample was approximately $10^{5} \mathrm{CFU} / \mathrm{mL}$. The results shown in Figure 5was as expected that the electrochemical signal obtained for other bacteria strains except Salmonella had no significantly difference with the control. While the mixture of all the four bacteria strains gave the similar signal with the sample just inoculated with Salmonella. The signal significantly different with the control was observed only in presence of the Salmonella. The result demonstrated that the strategy had a good specificity to Salmonella enteric.

\subsection{The reproducibility of the detection}

The reproducibility of this approach was studied by the detection of Salmonella inoculated in agricultural water from two different locations. Figure 6 shows the comparison of the electrochemical signal of the same concentration of Salmonella inoculated in the agricultural water. Three samples were analyzed and averaged for Salmonella samples in both agricultural waters at each concentration. Statistical significance between the agricultural water from two locations was determined using the analysis of variance with pooled-t-test. The results suggested that there was no significant difference between the two groups at each concentration, thus the method was reproducible between the samples $(\mathrm{P}<0.5)$. The threshold value to be claimed as Salmonella positive was estimated by the comparison of the mean value of negative control plus three standard deviations and the corresponding charge of the test sample.

\section{Conclusion}

A rapid electrochemical detection plus redox cycling combined with immunomagnetic concentration was developed for the detection of Salmonella. Two strategies of signal amplification were used in this approach that were attributed to immunomagnetic pre-concentration and redox cycling which based on the regeneration of AA from its oxidized product by TCEP. The enzymatic reaction is affected by incubation time, temperature and $\mathrm{pH}$ which can influence optimal electrochemical readings. Therefore, the condition of enzymatic reaction was optimized first. Under the optimal conditions: 30 minutes of incubation at $\mathrm{pH} 10,0$ and $40{ }^{\circ} \mathrm{C}$, the electrochemical detection was conducted to detect Salmonella inoculated in PBS buffer and agricultural water at relatively low concentrations. The combination of immunomagnetic pre-concentration and redox cycling allowed to detect Salmonella in less than 3 hours without a timeconsuming step of pre-enrichment and obtained a limit of detection of less than $10^{3} \mathrm{CFU} / \mathrm{mL}$. When the Salmonella was pre-concentrated for 4 hours, this method enabled detect as low as $10 \mathrm{CFU} / \mathrm{mL}$ of Salmonella. 
The ability to rapidly and efficiently determine the concentrations of potential pathogens will have a significant impact in the fields of food and agriculture. We have proposed a method which requires minimal instrumentation and resources. This strategy can also be applied to detect other bacteria in various food samples by incorporating different antibodies and appropriate preparation steps for the sample. Our goal was to develop an electrochemical assay due to the simplicity of instrumentation. The measurements can be conducted with a device with the complexity, size and cost of a common blood-glucose meter. The proposed method brings the potential of rapids field-deployable assays one step closer to reality.

\section{Acknowledgements:}

Support was provided by the Center for Hierarchical Manufacturing, a National Science Foundation Nanoscale Science and Engineering Center at the University of Massachusetts supported under Award Number CMMI-1025020and by the National Institute of Food and Agriculture, U.S. Department of Agriculture, the Massachusetts Agricultural Experiment Station and the Food Science Department of the University of Massachusetts Amherst, under project number MAS00440.

\section{References}

Akanda, M. R., Aziz, M. A., Jo, K., Tamilavan, V., Hyun, M. H., Kim, S., \& Yang, H. (2011). Optimization of Phosphatase- and Redox Cycling-Based Immunosensors and Its Application to Ultrasensitive Detection of Troponin I. Anal Chem, 83(10), 3926-3933.

Akanda, M. R., Tamilavan, V., Park, S., Jo, K., Hyun, M. H., \& Yang, H. (2013). Hydroquinone Diphosphate as a Phosphatase Substrate in Enzymatic Amplification Combined with Electrochemical-Chemical-Chemical Redox Cycling for the Detection of E. coli O157:H7. Anal Chem, 85(3), 1631-1636.

Anson, F. C., \& Osteryoung, R. A. (1983). CHRONOCOULOMETRY - A CONVENIENT, RAPID AND RELIABLE TECHNIQUE FOR DETECTION AND DETERMINATION OF ADSORBED REACTANTS. Journal of Chemical Education, 60(4), 293-296.

Arora, K., Chand, S., \& Malhotra, B. D. (2006). Recent developments in bio-molecular electronics techniques for food pathogens. Anal Chim Acta, 568(1-2), 259-274.

Chen, J., Alcaine, S. D., Jiang, Z., Rotello, V. M., \& Nugen, S. R. (2015). Detection of Escherichia coli in Drinking Water Using T7 Bacteriophage-Conjugated Magnetic Probe. Anal Chem, 87(17), 8977-8984.

Chen, J., Jiang, Z., Ackerman, J. D., Yazdani, M., Hou, S., Nugen, S. R., \& Rotello, V. M. (2015). Electrochemical nanoparticle-enzyme sensors for screening bacterial contamination in drinking water. Analyst, 140(15), 49914996.

Chen, J., Zhou, Y., Wang, D., He, F., Rotello, V. M., Carter, K. R., Watkins, J. J., \& Nugen, S. R. (2015). UVnanoimprint lithography as a tool to develop flexible microfluidic devices for electrochemical detection. Lab Chip, 15(14), 3086-3094.

Cho, I.-H., Mauer, L., \& Irudayaraj, J. (2014). In-situ fluorescent immunomagnetic multiplex detection of foodborne pathogens in very low numbers. Biosens Bioelectron, 57, 143-148.

Dean, R. L. (2002). Kinetic studies with alkaline phosphatase in the presence and absence of inhibitors and divalent cations. Biochemistry and Molecular Biology Education, 30(6), 401-407.

Goddard, J. M., \& Hotchkiss, J. H. (2007). Polymer surface modification for the attachment of bioactive compounds. Progress in Polymer Science, 32(7), 698-725.

Guosong Lai, F. Y., and Huangxian Ju. (2009). Dual signal amplification of glucose oxidase-functionalized nanocomposites as a trace label for ultrasensitive simultaneous multiplexed electrocehmical detection of tumor markers.pdf $>$.

Kessler, M. A. (1998). Determination of copper at ng ml(-1)-levels based on quenching of the europium chelate luminescence. Anal Chim Acta, 364(1-3), 125-129.

Kjems, L. L., Roder, M. E., Dinesen, B., Hartling, S. G., Jorgensen, P. N., \& Binder, C. (1993). Highly sensitive enzymeimmunoassay of proinsulin immunoreactivity with use of 2 monoclonal-antibodies. Clinical Chemistry, 39(10), 2146-2150. 
Kreuzer, M. P., O'Sullivan, C. K., \& Guilbault, G. G. (1999). Alkaline phosphatase as a label for immunoassay using amperometric detection with a variety of substrates and an optimal buffer system. Anal Chim Acta, 393(1-3), 95102.

Lee, K.-M., Runyon, M., Herrman, T. J., Phillips, R., \& Hsieh, J. (2015). Review of Salmonella detection and identification methods: Aspects of rapid emergency response and food safety. Food Control, 47, 264-276.

Liebana, S., Lermo, A., Campoy, S., Pilar Cortes, M., Alegret, S., \& Isabel Pividori, M. (2009). Rapid detection of Salmonella in milk by electrochemical magneto-immunosensing. Biosens Bioelectron, 25(2), 510-513.

Mazzei, F., Botre, F., Montilla, S., Pilloton, R., Podesta, E., \& Botre, C. (2004). Alkaline phosphatase inhibition based electrochemical sensors for the detection of pesticides. Journal of Electroanalytical Chemistry, 574(1), 95-100.

Nam, E. J., Kim, E. J., Wark, A. W., Rho, S., Kim, H., \& Lee, H. J. (2012). Highly sensitive electrochemical detection of proteins using aptamer-coated gold nanoparticles and surface enzyme reactions. Analyst, 137(9), 2011-2016.

Nugen, S. R., \& Baeumner, A. J. (2008). Trends and opportunities in food pathogen detection. Analytical and Bioanalytical Chemistry, 391(2), 451-454.

Park, S., \& Yang, H. (2014). Sensitive and selective trypsin detection using redox cycling in the presence of L-ascorbic acid. Analyst, 139(16), 4051-4055.

Shields, M. J., Hahn, K. R., Janzen, T. W., Goji, N., Thomas, M. C., Bin Kingombe, C. I., Paquet, C., Kell, A. J., \& Amoako, K. K. (2012). Immunomagnetic Capture of Bacillus anthracis Spores from Food. Journal of Food Protection, 75(7), 1243-1248.

Shim, W.-B., Song, J.-E., Mun, H., Chung, D.-H., \& Kim, M.-G. (2014). Rapid colorimetric detection of Salmonella typhimuriumusing a selective filtration technique combined with antibody-magnetic nanoparticle nanocomposites. Analytical and Bioanalytical Chemistry, 406(3), 859-866.

Singh, A., Park, S., Yang, H. (2013). Glucose-oxidase label-based redox cycling for an incubation period-free electrochemical immunosensor. Anal Chem, 85(10), 4863-4868.

Steingroewer, J., Knaus, H., Bley, T., \& Boschke, E. (2005). A rapid method for the pre-enrichment and detection of Salmonella typhimurium by immunomagnetic separation and subsequent fluorescence microscopical techniques. Engineering in Life Sciences, 5(3), 267-272.

Taskila, S., Tuomola, M., \& Ojamo, H. (2012). Enrichment cultivation in detection of food-borne Salmonella. Food Control, 26(2), 369-377.

Tatavarthy, A., Peak, K., Veguilla, W., Cutting, T., Harwood, V. J., Roberts, J., Amuso, P., Cattani, J., \& Cannons, A. (2009). An Accelerated Method for Isolation of Salmonella enterica Serotype Typhimurium from Artificially Contaminated Foods, Using a Short Preenrichment, Immunomagnetic Separation, and Xylose-LysineDesoxycholate Agar (61X Method). Journal of Food Protection, 72(3), 583-590.

Velusamy, V., Arshak, K., Korostynska, O., Oliwa, K., \& Adley, C. (2010). An overview of foodborne pathogen detection: In the perspective of biosensors. Biotechnol Adv, 28(2), 232-254.

Walter, A., Wu, J., Flechsig, G.-U., Haake, D. A., \& Wang, J. (2011). Redox cycling amplified electrochemical detection of DNA hybridization: Application to pathogen E. coli bacterial RNA. Anal Chim Acta, 689(1), 29-33.

Wang, H., Li, Y., Wang, A., \& Slavik, M. (2011). Rapid, Sensitive, and Simultaneous Detection of Three Foodborne Pathogens Using Magnetic Nanobead-Based Immunoseparation and Quantum Dot-Based Multiplex Immunoassay. Journal of Food Protection, 74(12), 2039-2047.

Wang, Y., Fill, C., \& Nugen, S. R. (2012). Development of chemiluminescent lateral flow assay for the detection of nucleic acids. Biosensors, 2(1), 32-42.

Wilson, M. S. R., R. D. (2004). Hydroquinone diphosphate: an alkaline phosphatase substrate that does not produce electrode fouling in electrochemical immunoassays. Biosens Bioelectron, 20(2), 276-283.

Wu, J., Yan, Y., Yan, F., \& Ju, H. (2008). Electric field-driven strategy for multiplexed detection of protein biomarkers using a disposable reagentless electrochemical immunosensor array. Anal Chem, 80(15), 6072-6077.

Xia, N., Ma, F., Zhao, F., He, Q., Du, J., Li, S., Chen, J., \& Liu, L. (2013). Comparing the performances of electrochemical sensors using p-aminophenol redox cycling by different reductants on gold electrodes modified with self-assembled monolayers. Electrochimica Acta, 109, 348-354.

Y. Liu, Y. C., Yanbin Liu. (2001). Rapid detection of Salmonella typhimurium using immunomagnetic separation and immuno-optical sensing method. Sensors and Actuators $B$.

Yang, H. (2012). Enzyme-based ultrasensitive electrochemical biosensors. Curr Opin Chem Biol, 16(3-4), 422-428.

Yang, L. J., Ruan, C. M., \& Li, Y. B. (2001). Rapid detection of Salmonella typhimurium in food samples using a bienzyme electrochemical biosensor with flow injection. Journal of Rapid Methods and Automation in Microbiology, 9(4), 229-240. 
Yeni, F., Acar, S., Polat, O. G., Soyer, Y., \& Alpas, H. (2014). Rapid and standardized methods for detection of foodborne pathogens and mycotoxins on fresh produce. Food Control, 40, 359-367.

Zheng, Q., Mikg-Krajnik, M., Yang, Y., Xu, W., \& Yuk, H.-G. (2014). Real-time PCR method combined with immunomagnetic separation for detecting healthy and heat-injured Salmonella Typhimurium on raw duck wings. International Journal of Food Microbiology, 186, 6-13.

394 


\section{Figure1}

(a)

Magnetic beads coated with anti-Salmonella antibodies
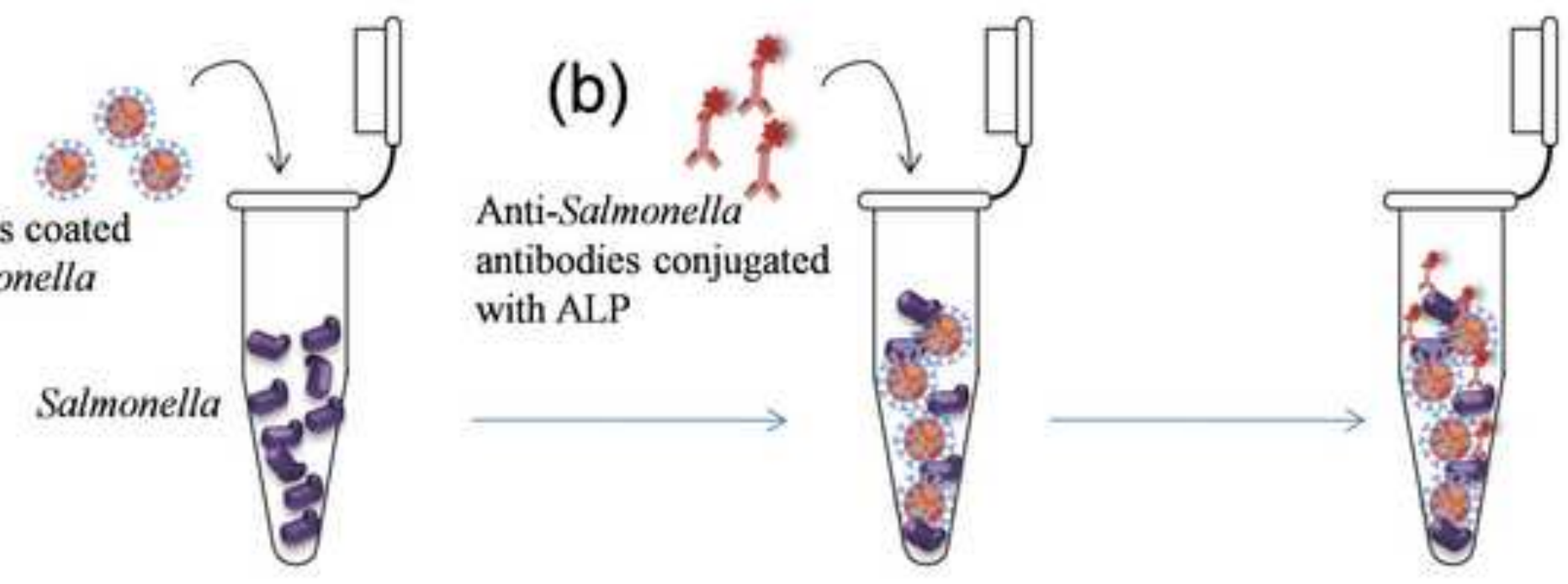

(c)
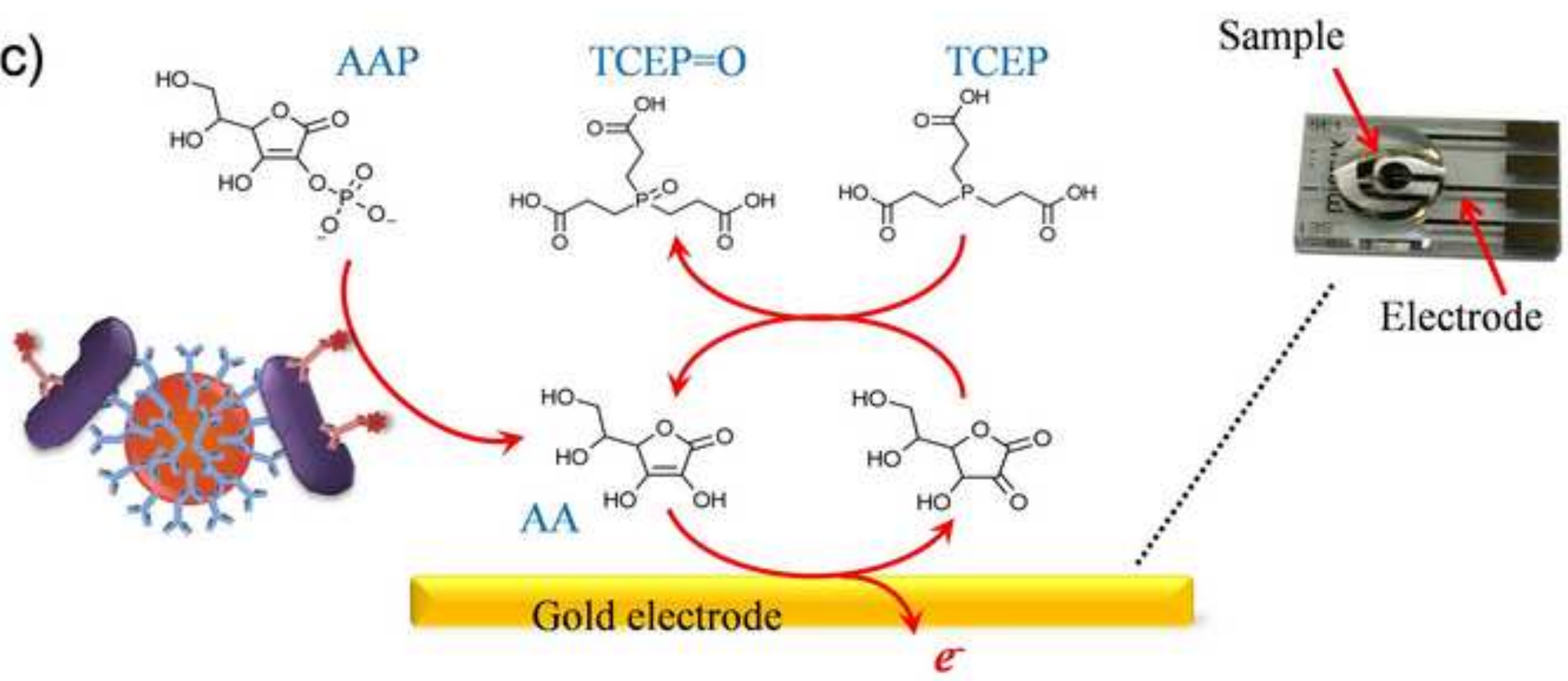

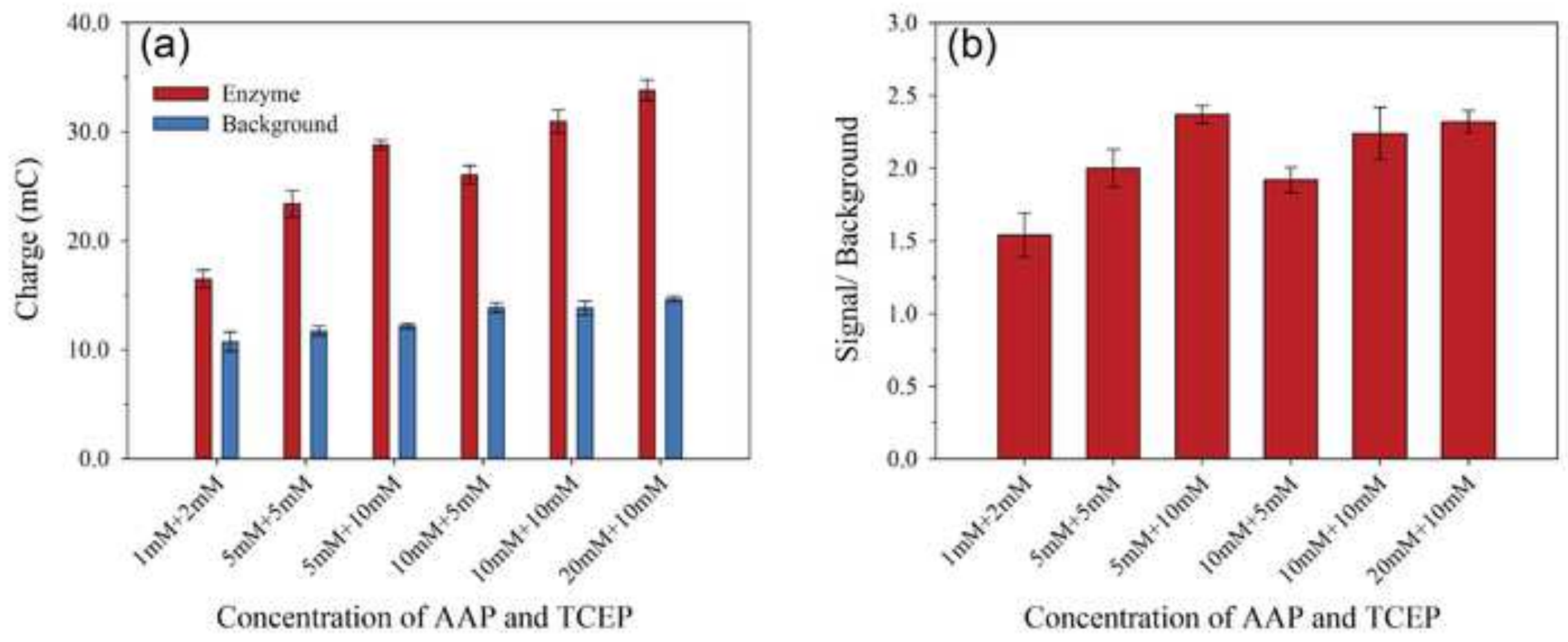

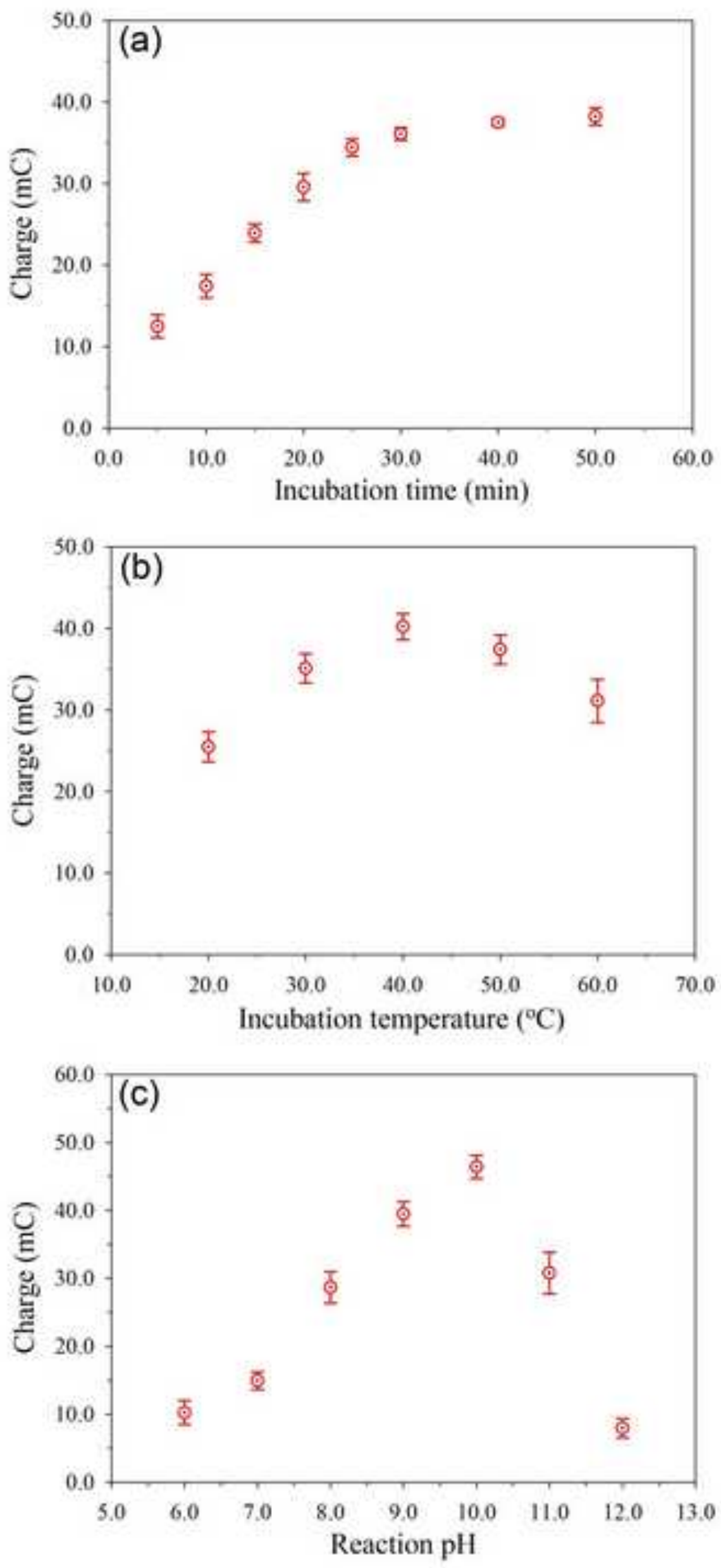

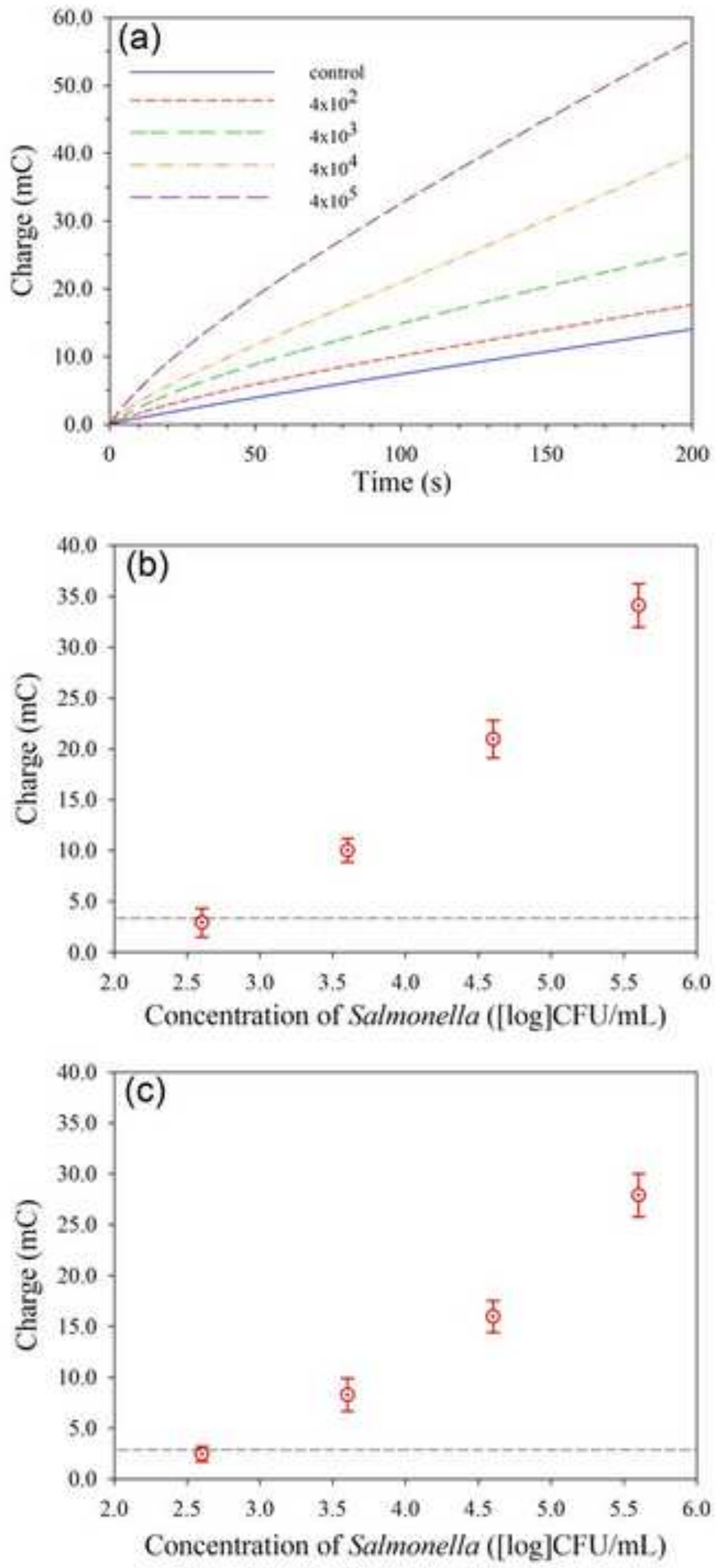


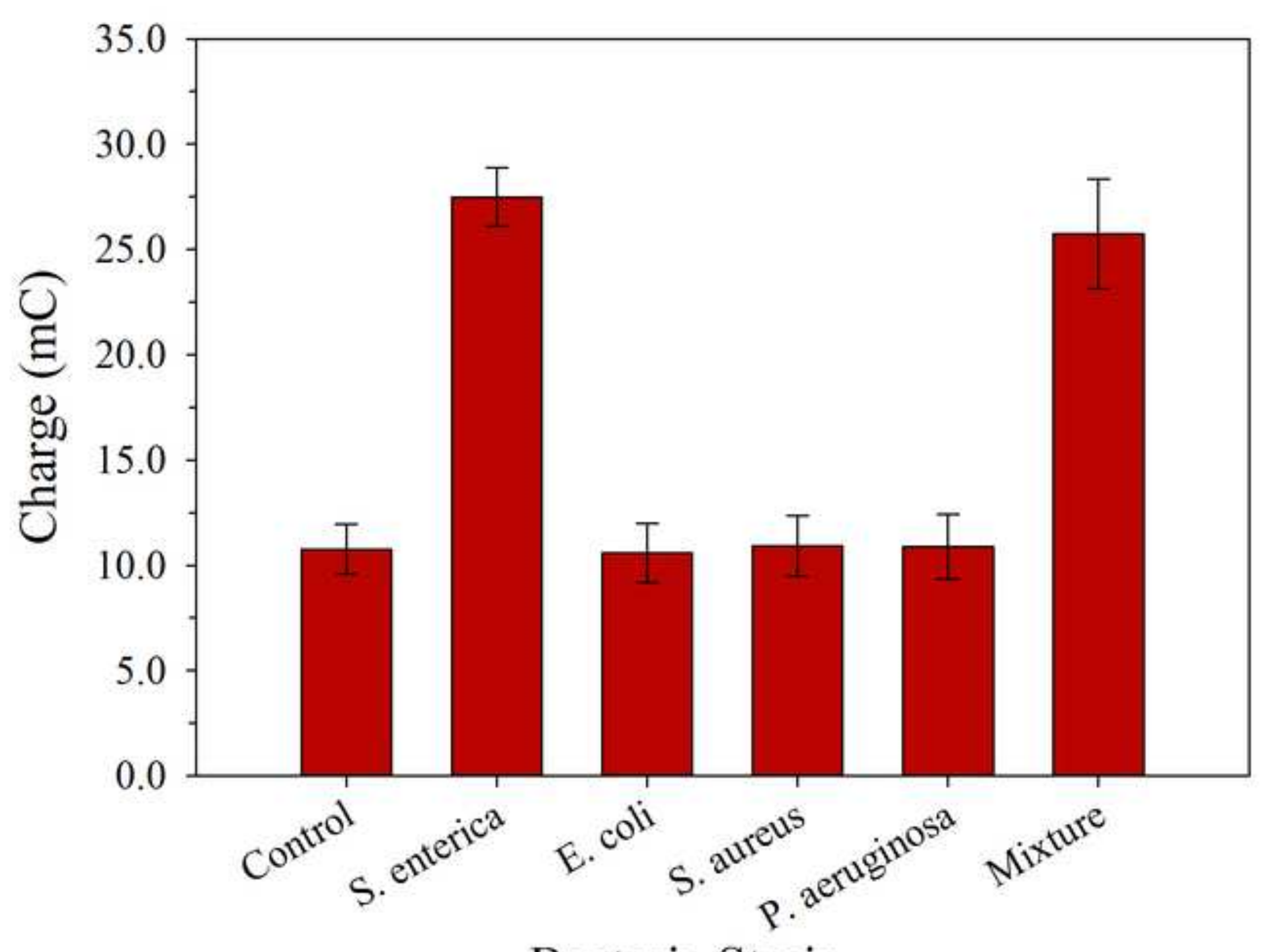

Bacteria Strain 


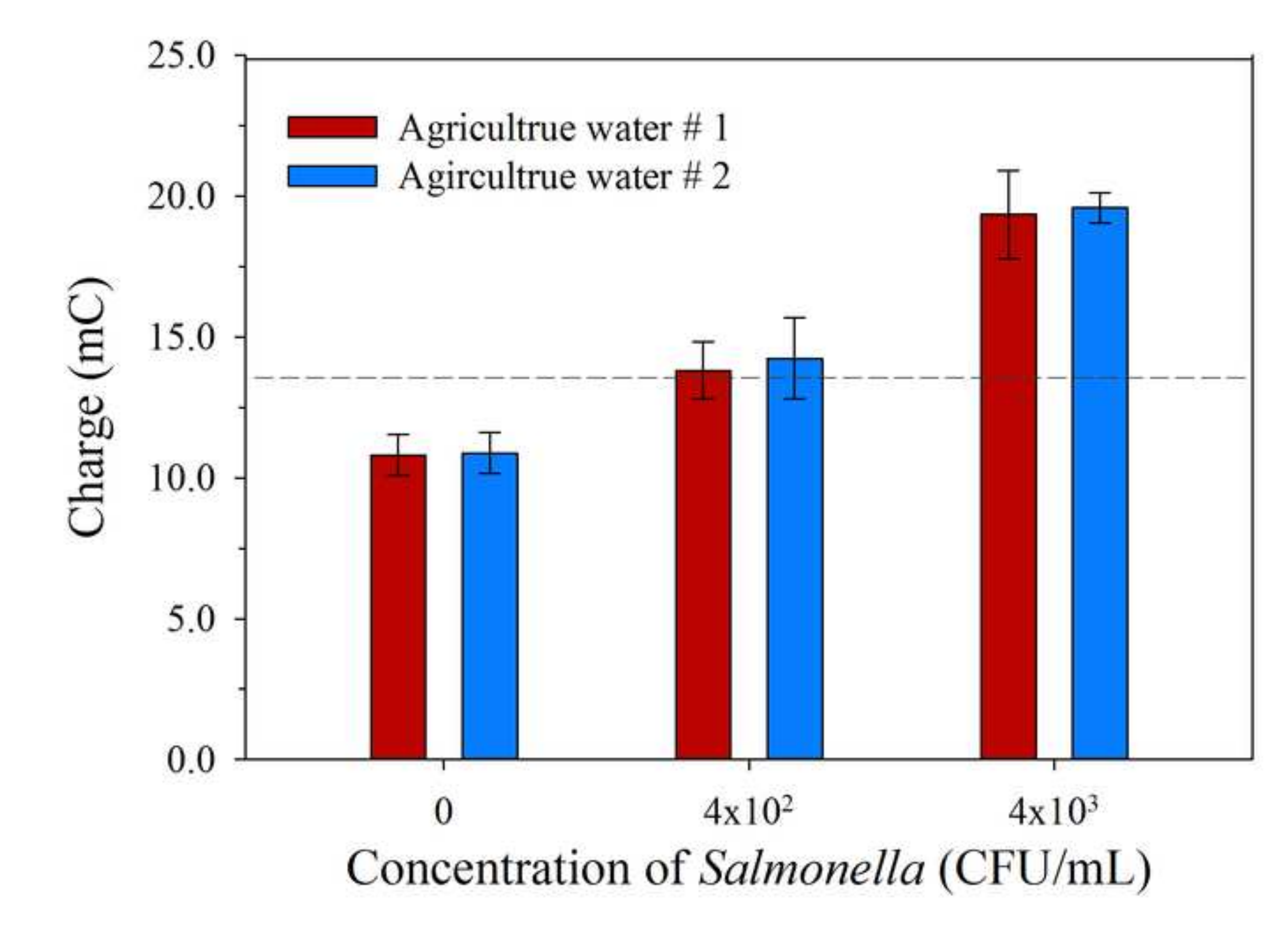

Concentration of Salmonella (CFU/mL)

.


Figure 1 Schematic representation of the immunomagnetic pre-concentration and electrochemical detection based on redox cycling. The detection procedure contained three main steps. (a) Immunomagnetic separation and pre-concentration of Salmonella from sample matrix. (b) Immunological reaction with anti-Salmonella antibodies conjugated with alkaline phosphatase. (c) Enzyme reaction and electrochemical detection.

Figure 2 (a) Charge obtained for varying concentration of AAP and TCEP with and without enzyme reaction (background) at room temperature for 20 minutes. (b) The ratio of the charge obtained after enzyme reaction over the background. The error bars represent the standard deviation of three measurements.

Figure 3 Optimization results of incubation time, temperature and $\mathrm{pH}$ of the enzymatic reaction. (a) Incubation time dependence of the charge at room temperature at $\mathrm{pH}$ 8.9. (b) Dependence of the charge on incubation temperature after a 30 minutes incubation at $\mathrm{pH}$ 8.9. (c) Dependence of the charge on the reaction $\mathrm{pH}$ after a 30 minutes incubation at $40^{\circ} \mathrm{C}$

Figure 4 Results of electrochemical detection (a) Choronocoulograms (charge dependence of time) that were obtained at a potential of $0.6 \mathrm{~V}$ on a gold electrode for Salmonella with a concentration of $4 \times 10^{2}$, $4 \times 10^{3}, 4 \times 10^{4}, 4 \times 10^{5} \mathrm{CFU} / \mathrm{mL}$ in $1 \times \mathrm{PBS}$ buffer after adjusting the $\mathrm{pH}$ of reaction solution to 10.0 and incubating for $30 \mathrm{~min}$ at $40{ }^{\circ} \mathrm{C}$. Dependence of the charge at $150 \mathrm{~s}$ on the concentration of Salmonella artificially inoculated in (b) $1 \times$ PBS buffer and (c) agricultural water. The dash line represents three times of the standard deviation (SD) of the signal at $0 \mathrm{CFU} / \mathrm{mL}$. The error bars correspond to the standard deviation of nine replicates. Each point was averaged by nine measurements.

Figure 5 Electrochemical signal obtained for the specificity study. The bars show that the charge for agricultural water inoculated with Salmonella, E. coli, S. aureus, P. aeruginosa and a mixture of each strains, respectively. The error bars correspond to the standard deviation of three replicates.

Figure 6 The comparison of the electrochemical detection results of Salmonella inoculated in agricultural water from two different locations. The data represent the electrochemical signal obtained at $150 \mathrm{~s}$ from the chronocoulograms for the two agricultural water, respectively, inoculated with $0 \mathrm{CFU} / \mathrm{mL}$ (negative control), $4 \times 10^{2} \mathrm{CFU} / \mathrm{mL}$, and $4 \times 10^{3} \mathrm{CFU} / \mathrm{mL}$ of Salmonella. The dashed line represents three times of the standard deviation (SD) of the signal at $0 \mathrm{CFU} / \mathrm{mL}$ plus the signal of the control $(0 \mathrm{CFU} / \mathrm{mL})$. The error bars represent the standard deviation of three measurements. 